\title{
A Directional Selective Power Routing Protocol for the Internet of Underwater Things
}

\author{
Manal Al-Bzoor $\mathbb{D}^{1},{ }^{1}$ Ahmed Musa $\left(\mathbb{D},{ }^{2}\right.$ Khawla Alzoubi $\mathbb{D}^{3},{ }^{3}$ and Taha Gharaibeh $\mathbb{D}^{1}$ \\ ${ }^{1}$ Department of Computer Engineering, Yarmouk University, Irbid, Jordan \\ ${ }^{2}$ Department of Communication Engineering, Yarmouk University, Irbid, Jordan \\ ${ }^{3}$ Department of Engineering Technology, Community College of Qatar, Doha, Qatar \\ Correspondence should be addressed to Manal Al-Bzoor; mbzoor@yu.edu.jo
}

Received 21 December 2021; Revised 14 January 2022; Accepted 17 January 2022; Published 8 February 2022

Academic Editor: Hamada Esmaiel

Copyright (c) 2022 Manal Al-Bzoor et al. This is an open access article distributed under the Creative Commons Attribution License, which permits unrestricted use, distribution, and reproduction in any medium, provided the original work is properly cited.

\begin{abstract}
The Internet of Underwater Things (IoUT) has lately gained popularity as a means of facilitating a wide range of underwater applications. In the IoUT, underwater communication is best accomplished by the usage of acoustic waves, whereas the terrestrial communication between the surface sinks and the on-shore control stations is typically achieved using radio waves. As a result, the greatest portion of an IoUT is enabled by the underwater acoustic sensor network (UASN), where the specific issues provided by the use of acoustic waves, the underwater node mobility, and the localization difficulties have yet to be addressed. In this paper, we discuss the challenges faced by the IoUT in terms of the currently proposed routing protocols and propose a Directional Selective Power Routing Protocol (DSPR) to cope with most of these challenges. The proposed protocol (i.e., DSPR) uses the angle of arrival and the sender depth information to find the best direction to the surface sink. In addition, the DSPR uses selective power control to enhance the delivery ratio and ensure connectivity while reducing energy consumption. To testify the performance of the proposed protocol, intensive simulation experiments have been conducted. The simulation results show that the proposed DSPR protocol outperforms two variations of the fixed directional routing (DR) protocol and the variable power depth-based routing (VDBR) protocol in terms of energy consumption and delivery ratio. For instance, the proposed DSPR protocol achieves at least 8 times enhancement in energy consumption compared with VDBR. In addition, DSPR saves around 30\% of energy consumption over the DR protocols when the network is mobile. Moreover, the DSPR protocol acquires a delivery ratio above $90 \%$ for static/ dynamic scenarios in both sparse and dense networks.
\end{abstract}

\section{Introduction}

Recent years have witnessed a rise in the usage of Internet of things (IoT), where smart devices from different heterogeneous systems are connected to exchange data and form one homogeneous large system. The IoTs have been extended to include the underwater networks deployed in submarine environment such as oceans, seas, etc. This extension is uniquely identified as the Internet of underwater things (IoUT). The IoUT is a promising technology that connects around $72 \%$ of the Earth surface with the land-connected Internet. In addition, this promising technology will serve many potential applications such as environmental monitoring, undersea explorations, disaster prevention, military submarine tracking, and oil industry [1]. These applications require the deployment of smart underwater system which uses a mishmash of acoustic, radio, and optical waves for communications. It is worth mentioning that the optical waves suffer sever attenuation in the underwater environment. Henceforth, it can only be utilized at extremely high frequencies in the range of $\mathrm{THz}$ or in shallow water applications. Furthermore, the optical waves mandate a clear and precise Line of Sight (LOS), which is hard to achieve in marine environment [2]. In addition, the optical wave characteristics varies according to the water content, the temporal changes produced by turbulence, and other environmental variables. However, the underwater radio communication often operates at frequencies ranging from a 
few $\mathrm{kHz}$ to hundreds of $\mathrm{MHz}$, providing high data rates and low propagation delays [3]. Nevertheless, the radio waves suffer from significant attenuation at high frequencies and require the use of large and expensive antennas at low frequencies. As a result, the radio waves have been investigated in the communication between the surface sinks, buoys, and the offshore infrastructure. Additionally, the radio waves have been utilized in the shallow regions for real-time underwater applications, which require short latency and large bandwidth. Another communication technology called magneto inductive (MI) has been proposed as a carrier for data communication in underwater wireless sensor networks. Albeit the MI technology does not suffer from multipath fading, scattering, and signal propagation delay, it is only efficient for shallow water applications at distances up to $40 \mathrm{~m} \mathrm{[4]}$.

According to the aforementioned limitations of radio, optical, and MI systems, acoustic waves are extensively employed as an alternative carrier for data communication in deep underwater environments. The key characteristics of acoustic waves include the low attenuation that allows signals to travel long distances without losing data. However, the main drawback of acoustic waves is long propagation delays and limited bandwidth. Fortunately, most underwater networks are used to send small sized packets to the surface or to the on-land Internet that represent the sensory data. Thus, the limited bandwidth imposed by the acoustic waves is no longer a significant communication barrier.

The key contribution of this work is twofold. Firstly, we highlight the challenges imposed by acoustic waves usage in IoUT communication. In addition, we discourse the effect of underwater nodes mobility patterns on the design of successful routing protocols $[5,6]$. Secondly, we propose a reliable and energy-efficient routing protocol named directional selective power (DSPR) to combat the challenges of IoUT. In the DSPR protocol, the underwater nodes do not need the three-dimensional position of themselves or other nodes within the network. The underwater nodes communicate their sensed data in the direction of the nearest sink by employing the arrival angle of the acoustic signal $[7,8]$. Furthermore, we combine the directional approach by a selective power control to maintain the network connectivity [9]. Thus, we increase the delivery ratio and enhance the overall network reliability. Moreover, the DSPR protocol is applicable for both sparse and dense networks as it can cope with the mobility of underwater nodes.

The rest of this paper is organized as follows. Section 2 presents the main features of the Internet of underwater things and the key issues that result from the acoustic wave utilization, the nodes mobility, and the difficulty of localization within the three-dimensional underwater architecture. Section 3 summarizes the main locations unaware routing techniques used for underwater acoustic sensor network (UASN) and the IoUT. Section 4 presents and analyzes the proposed routing protocol.Section 5 presents and illustrates the simulation results. Finally, Section 6 concludes the paper and gives directions for future work.

\section{Challenges for Efficient Communication in Internet of Underwater Things}

As was previously mentioned, an IoUT is enabled mainly by the use of UASN. In this type of network, smart underwater sensor nodes, autonomous underwater vehicles (AUV), and remotely operated vehicles (ROV) have been introduced to the research and industry communities to facilitate various underwater applications [10]. The smart underwater sensor nodes are often equipped with several types of sensors, an acoustic modem, and other smart features. These nodes may record various observed data and selectively send them to a surface sink. In general, the surface sink is equipped with both acoustic and radio modems to connect the undersea world to the terrestrial Internet and hence forms the IoUT. Figure 1 illustrates an example of an IoUT where acoustic wireless links are used as a communication medium between a surface sink, a set of underwater nodes, and an autonomous underwater vehicle [1]. The communicated data are then relayed by the surface sink to a satellite station or to onshore control centers using radio links. The upcoming subsections present the challenges and crucial issues for efficient communication in the IoUT.

Like IoT, the performance metrics such as reduced energy consumption, reliability, and shorter end-to-end delays are the main concerns for designers of the IoUT protocols. Unfortunately, there are different communication challenges in the underwater environment than that of the terrestrial environment. Therefor, to improve the overall network performance, different performance and special design factors are to be considered. These metrics include the unique characteristics of acoustic waves, the deployment architecture, the high energy requirements, the mobility of underwater nodes, and the localization difficulty $[1,11]$. Table 1 compares the unique characteristics and challenges experienced by the designers of IoUT with those experienced by IoT. The next subsections elaborate more on each of these challenges.

2.1. Acoustic Wave Communication. The acoustic waves compromise the largest part of communications in the underwater world. The radio waves are still the dominant medium for communication between surface sinks and the on-land control stations. Needless to say, the acoustic waves have their own set of challenges, such as the limited bandwidth and the slow propagation speed $(1500 \mathrm{~m} / \mathrm{s})$. This speed is five times slower than that of radio waves. The acoustic signal speed is also affected by different water characteristics such as salinity, depth, and temperature. The effect of these characteristics is more noticeable near the shore or in the shallow water, where salinity and temperature vary greatly. In deep oceans, these variations are limited and hence the acoustic signal speed is almost fixed. The depth of water is another factor that impacts the sound speed where it increases by the increase in water pressure. Therefore, in deep underwater communications where the acoustic waves are required to travel for distances up to a few kilometers, the slow speed of sound results in huge 


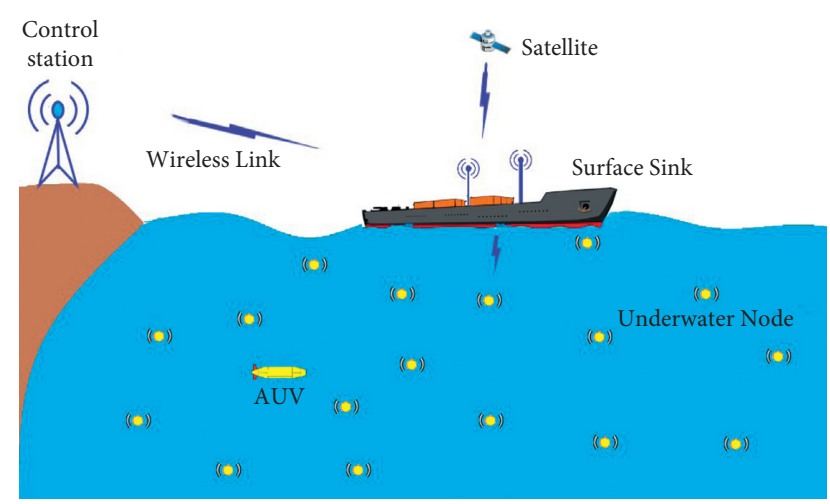

FIgURe 1: An architecture for IoUT.

TABle 1: IoUT vs IoT features and challenges.

\begin{tabular}{|c|c|c|}
\hline Feature & IoUT & IoT \\
\hline $\begin{array}{l}\text { Communication } \\
\text { medium }\end{array}$ & Acoustic waves & Radio wave \\
\hline Propagation speed & $1500 \mathrm{~m} / \mathrm{s}$ & $2 \times 10^{8} \mathrm{~m} / \mathrm{s}$ \\
\hline Transmission range & $100-10000$ meters & $10-100$ meters \\
\hline Localization & Expensive algorithms & Cheap with GPS \\
\hline Modem & $\begin{array}{c}\text { Expensive large/ } \\
\text { acoustic }\end{array}$ & $\begin{array}{l}\text { Cheap small/ } \\
\text { radio }\end{array}$ \\
\hline Transmission power & Up to $10 \mathrm{~s}$ of watts & In milliwatts \\
\hline Data rate & In 10 's of kbps & In Mbps \\
\hline $\begin{array}{l}\text { Deployment } \\
\text { architecture }\end{array}$ & Mostly 3D & Mostly 2D \\
\hline Mobility & $\begin{array}{c}\text { Affected by water } \\
\text { current }\end{array}$ & Easily tracked \\
\hline
\end{tabular}

propagation delays $[3,12]$. Hence, for time sensitive IoUT applications, optimized delivery techniques in the presence of such long delays are required. It is either done by reducing the control packet exchange in multihop routing or by using one or more AUVs for data collection in a timely manner [13].

The acoustic waves suffer from absorption loss that is directly affected by the imperfections of the water medium, the frequency used by acoustic transceivers, and the distance traveled by the acoustics signal. The absorption loss of both radio and optical waves is much higher than that for acoustic waves [12]. Moreover, the multipath propagation can severely affect the acoustic based communication in under water medium. For instance, in the shallow water environment, the multipath arrivals of reflected signals are caused by the surface or bottom of the deployment region. In deep water, the multipath propagation can occur due to the refraction induced by changing the acoustic wave speed [3]. These challenges are usually overcome by the modem designs according to the physical layer level. However, the effect of multipath propagation may be mitigated at other protocol layers [14].

2.2. Deployment Architecture and Energy Efficiency. The deployment architecture of underwater sensor networks is inextricably coupled with the application requirements. For example, in some applications, the sensor nodes are deployed in a two-dimensional centralized topology at the seabed. A highly efficient node (gateways) collects data from sensor nodes and forwards it to the surface sink directly via a single hop. Other applications might use multihop communication to interact with one or more surface sinks that require the deployment of sensor nodes in a three-dimensional architecture at various depths. This will lead to categorizing the UASN deployments into either a two-dimensional or a three-dimensional architecture. The deployment strategies can also be classified as static if it is performed once at the network setup or dynamic/ adaptive if a redeployment decision is made during the network operation. Pompili et al. in [15] discussed the two architectures and investigated the problem of achieving maximal coverage with the lowest sensor count. These architectures were combined with multiple sink architecture to efficiently reduce delay, decrease energy consumption, and alleviate the limited bandwidth problem in acoustic communications [16]. The static deployment was illustrated by many frameworks and thus, many solutions were proposed for the underwater sensor network deployment. The dynamic node deployment strategies, on the other hand, are required to encounter the mobility of underwater nodes $[11,17,18]$. Nevertheless, dynamic deployment necessitates physical relocation of nodes and continuous update of nodes positions. Therefore, the mobility handling at the protocol layer stack is less expensive than dealing with it via dynamic deployment.

It is worth noting that the underwater nodes are battery powered. Henceforth, conserving their energy is a crucial requirement. This requirement has risen since most underwater node' deployment is done in remote underwater regions. Thus, replacing the battery of these nodes is difficult or infeasible. Furthermore, the acoustic modems require higher power than the radio modems, which can be in the order of watts compared to milliwatts for the radio modems. As a result, an underwater network protocol should prioritize energy efficiency while maintaining reliable connectivity.

2.3. Mobility and Localization. In the underwater settings, the node movement is unavoidable, negating the benefits of static-based methods proposed for terrestrial WSN. As a result, resilience to mobility should always be considered while designing efficient routing protocols [19]. In the AUVs and remotely operated vehicles (ROVs), mobility is supervised and controlled. On the other hand, the floating underwater sensor nodes are affected by uncontrolled mobility due to the water currents and dispersion that is also influenced by water temperature and varies with depth. In the UASN, simulating and predicting the performance of a given protocol requires precise modeling of nodes' mobility. This is because the node movement in a particular undersea environment is not completely random. Thus, customized mobility models such as the meandering model and the tidal mobility model have been used for modeling the movement of the underwater nodes $[20,21]$. The tidal model is better suited in offshore locations where tides are the primary 
driver of water movement, whereas the meandering one is used in the deepwater environments. Nonetheless, more precise prediction models based on the spatial and temporal correlation of movement patterns in UASN are still needed [22-25].

When compared to other routing categories, geographicbased routing protocols have been found to be the most scalable and efficient for UASN [26]. Nevertheless, the majority of these protocols need that nodes be aware of their three-dimensional position [27]. However, in the presence of mobility, locating nodes in large-scale mobile underwater sensor networks is a challenge. This is because the typical GPS-based approaches cannot operate in the water medium. Therefore, the localization is typically accomplished using expensive algorithms, which might cost additional energy expenditure and time $[6,28]$. As a result, location unaware routing protocols are favored by the UASN.

\section{Routing Protocols for the IoUT}

According to the aforementioned challenges of underwater communication, the location unaware routing protocols are best suited for communication in the underwater part of the IoUT network. There have been various efficient location unaware routing protocols proposed [29]. However, only a few discussed the successful data delivery while maintaining the network connectivity and saving energy expenditure in the presence of nodes mobility in the IoUT communication.

One of the location unaware routing protocols proposed for underwater communication is depth-based routing (DBR) [30]. In the DBR protocol, the depth information collected by the pressure sensors is the only dimension used by the network nodes for data forwarding. The nodes forward data if the depth of the current node is less than that of the previous node. The DBR protocol improves the performance of the dynamic scenarios. However, it still suffers from void region handling and redundant forwarding with high energy consumption. To overcome the problem of energy efficiency in the DBR protocol, an Enhanced Energy Depth-Based Routing (EEDBR) protocol was proposed in [31]. In this protocol, a residual energy indicator is used to achieve energy balancing and efficiency. The authors of [32] proposed two variants to enhance the DBR protocol. The first variation (or protocol) is an energy efficient one that is developed to decrease the redundancy of forwarding in the multiple paths. The second variation is the directional depth which is developed to enhance the end-to-end delay. In the first protocol, energy efficiency is achieved by prohibiting the nodes in the void region from performing the forwarding process. Instead, these nodes use the time of arrival ranging technique to decide on nodes in void regions. Surely, this will add extra overhead. In the directional DBR protocol, the nodes use the depth and angle metrics in the holding time calculations to guide the packets forwarding toward a surface sink via an optimal route. Thus, the end-to-end delays are drastically decreased and the delivery ratio is enhanced as well. However, in both protocols, the redundancy is reduced but not eliminated and void region formation is handled via expensive ranging technique in only one enhancement of the DBR protocol.

In [33], Adaptive Mobility of Courier nodes in Threshold-Optimized DBR (AMCTD) protocol is proposed. To reduce the number of forwarders, the AMCTD sets a predefined depth threshold for numerous nodes based on the network density. In addition, the AMCTD relies on a motorized movement of a set of courier nodes to handle void regions. Therefore, this protocol was not appropriate for data-sensitive applications. Hence, an Improved-AMCTD (I-AMCTD) protocol is developed in [34]. The I-AMCTD protocol minimizes the end-to-end delay by optimizing the mobility pattern of the sink. It also varies the depth threshold in order to increase the number of forwarders to minimize data loss and enhance the delivery ratio for delay-sensitive applications. However, like the AMCTD, the locations of courier nodes are mechanically adjusted and thus, extra overhead will take place.

A Delay-Sensitive Depth-Based Routing (DSDBR) protocol was proposed to solve the delay problem in the depth based routing schemes [35]. Here, the authors implement a delay sensitive holding time and a delay efficient priority factor. These factors reduce the end-to-end delay at an expense of lowering the throughput. Unfortunately, the DSDBR protocol was demonstrated only for static scenarios. In these scenarios, a time-consuming mechanical movement of courier nodes is used to bypass the void region.

Moreover, an Enhanced location un-aware Channel Aware Routing Protocol (ECARP) was specifically proposed for the IoUT [36]. This protocol is an enhancement of the channel aware routing protocol [37]. The ECARP protocol uses the previously collected information to aid forwarders in the decision whether to forward the incoming packets or not. Forwarders in ECARP might select a previous-hop forwarder even if this hop is not the best. This selection is performed to cope with the mobility of the nodes. This protocol efficiently forwards useful packets and reduces energy consumption. In ECARP, the nodes are assumed to store sensory data and make a comparison before forwarding decisions. This comparison will add extra storage cost and processing overhead. Furthermore, the network nodes are expected to switch between two power levels to achieve the same bit error ratio for both control and data packets.

A localization-Free Interference and Energy Holes Minimization (LF-IEHM) routing protocol was proposed to overcome the energy hole problem in multihop routing [38]. The authors of [38] used the holding time to limit the interference between consecutive Hello packets for forwarders discovery. Further, a variable transmission range is used by the sensor node to select forwarders. However, this selection process relies on the sound pressure value. According to this value, a forwarder that is not in the direction of the surface sink might be selected. In addition, it is not clear how the transmission range of nodes varies. Needless to say, this range should be directly related to the acoustic modem power.

In [8], a shortest path routing protocol based on the vertical angle (SPRVA) is proposed. This protocol improves 
both energy efficiency and the end-to-end delay by using the directional approach. This protocol is similar to the protocol we proposed as both use the directional approach. In the SPRVA protocol, the best forwarder is chosen according to the main priority which is indicated by the residual energy and the vertical angle between the propagation and the depth orientations. An alternative priority is utilized when the main priorities of the candidate nodes are equal. Reduced delivery ration in a sparse deployment is mitigated by implementing a recovery algorithm to bypass the nodes in void regions from participating in the forwarding process.

In an energy efficient routing approach for the IoUTs, researchers have used terrestrial based adaptive clustering protocols to enhance the delivery ratio and to reduce the energy consumption [39]. Here, an extension of a lifetime is achieved compared with the depth based routing. In general, the clustering approach provides energy balance, but it comes at a high cost in terms of communication overhead, especially, in mobile scenarios.

Authors in [40] introduced an opportunistic routing for optoacoustic IoUTs. They used the directional nature of light to guide the packet forwarding toward the surface sink. Unlike our proposed protocol, this protocol is applicable only for shallow and clear underwater communication.

The most recent proposed enhancement for the DBR protocol suggests using the variable power control protocol to bypass the routing voids [41]. However, the high energy consumption of this protocol has not been solved yet.

Taking routing decisions based on predicting mobility is proposed in $[25,42,43]$. However, in order to reduce the prediction error and obtain high efficiency, a huge input data should be recorded and analyzed by time consuming algorithms.

In our work, we consider the unique challenges of underwater environment while focusing on the application requirements for IoUTs. These requirements include increasing the delivery ratio and reducing the energy expenditure in both dense and sparse deployment. The proposed protocol is location unaware. In addition, it combines the directional and the selective power routing approaches in a three-dimensional mobile network to handle the void regions and maintain the connectivity.

\section{A Directional Selective Power Routing Protocol for IoUT}

The multihop routing protocols are the best candidate for an Underwater acoustic sensor network. They are usually developed based on geographical information [5]. In this kind of routing protocol, the exact location of nodes is a requirement. However, in the underwater environment, nodes location is obtained by running costly localization techniques [6]. The cost grows exponentially when the process of finding a location is frequently performed. For instance, in a dynamic environment, the nodes are susceptible to constant movement with water currents and thus finding their exact location at a certain moment seems difficult. In the proposed location unaware directional selective power routing the network connectivity is maintained by using the power control and an optimized shortest path is selected based on the angle of arrival of acoustic signal.

4.1. Network Architecture. In the proposed protocol, we assume that an underwater network consists of one or $N$ surface sinks: $S_{1}, S_{2}, \ldots, S_{N}$. The surface sinks and the underwater nodes are both assumed to be equipped with an acoustic modem that is capable of switching to a set of $k$ power levels. For instance, the power level of node $i$ can be characterized as $P l_{i}^{t} \in 1,2, \ldots, k$. At each power level, the acoustic signal can be correctly received and detected within a range specified by the acoustic modem characteristics and channel equations. All nodes are equipped with on-board arrays of multiple microphones to determine the arrival angle of the received acoustic signal [44].

The proposed routing protocol is subdivided into two phases; (1) the network setup phase where nodes assign themselves to a certain sink and select the minimum power level to choose a set of possible forwarders. (2) The network operation phase where the nodes use the initial settings and can switch back to renew their possible forwarders if the network is disconnected due to mobility or node failure.

4.2. Network Setup. During network setup, the sink node will broadcast a Hello packet using its maximum transmission power. A surface sink is usually equipped with an acoustic transmitter that can send at maximum transmission power to a distance of up to a few kilometers. The underwater nodes which received the Hello packet will record the angle of arrival (sinkAoA) of each received sink's along with its sinkID. Using this information, the underwater nodes will determine the relative directions toward each of the surface sinks. Consequently, the underwater nodes will use this information to determine the directional path toward the closest sink $S_{i}$ that has the lowest vertical angle of arrival such as

$$
S_{i}=\underset{(t)}{\arg \min }\left\|\theta_{i}-90\right\| .
$$

Figure 2 shows an illustration of the sink selection process. Here, a source node $S$ receives two Hello packets from sink1 and sink2. Accordingly, it will select sink1 as its final destination since it has a lower arrival angle (i.e., $\theta_{1}<\theta_{2}$ ).

4.3. Forwarder Discovery. The forwarder discovery step is performed initially after the network setup. This step will be repeated when a sender receive no acknowledgments (Acks) for data packets due to the network topology change. In this step, the sender nodes send Hello packets to select the best forwarders using their minimum transmission power and sets a timer to receive the acknowledgments. The timer is selected to slightly exceed the round trip time required for the acoustic signal to reach the furthest node in its range. The waiting time $\left(w t_{i}\right)$ for each power level is defined by the following: 


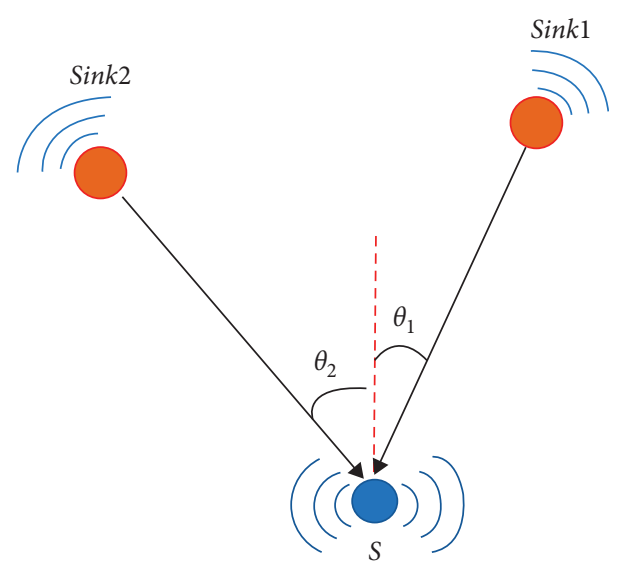

FIgURE 2: The source node $S$ selects its destination as sink1.

$$
w t_{i}=\frac{2 R_{i}}{c}+\delta
$$

where $R_{i}$ is the maximum distance at which an acoustic signal at a power level $\mathrm{Pl}_{i}$ can be correctly received and detected, $c$ is the sound speed $(1500 \mathrm{~m} / \mathrm{s})$, and $\delta$ is a marginal time added to slightly exceed the Hello packet transmission time.

Nodes receiving this Hello packet will reply with an Ack packet that includes the potential forwarder ID, its sin$\mathrm{kAoA}$, and residual energy level. Each forwarder holds an indicator to the residual energy level. These levels range from 1 to 5 where 1 indicates the least power level, whereas 5 indicates the maximum. A sender node will calculate the Ack angle of arrival (ackAoA) and compare it with its sinkAoA. The senders will select the best possible forwarders based on two metrics: the angle of arrival difference for the Ack packet and the sink identification packet and the residual energy level of potential forwarders. If no Ack is received before the timer expires, the sender switch to a higher power level to find forwarders and will again select the best forwarders based on sinkAoA and ackAoA. This step is repeated until the nodes find the correct power level needed to find the candidate forwarders or until the maximum power level is reached. Figure 3 shows a sender $S$ finds two possible forwarders when switching to the power level associated with range $r 2$. $S$ will then select between $n 1$ and $n 2$ based on the angle of arrival values and the residual energy level. It is worth mentioning that nodes will be disconnected if it cannot find neighbors at the highest available power level. Therefore, the number of power levels should be carefully selected.

4.4. Forwarder Selection. After the forwarder discovery step, each sender will maintain a table of possible forwarders with information extracted from the Ack packet including the forwarder ID, its ackAoA, and a residual energy indicator. The later information consists of multiple values where 1 and 5 values indicate very low and very high, respectively. When a sender has data to send, it will only look for forwarders

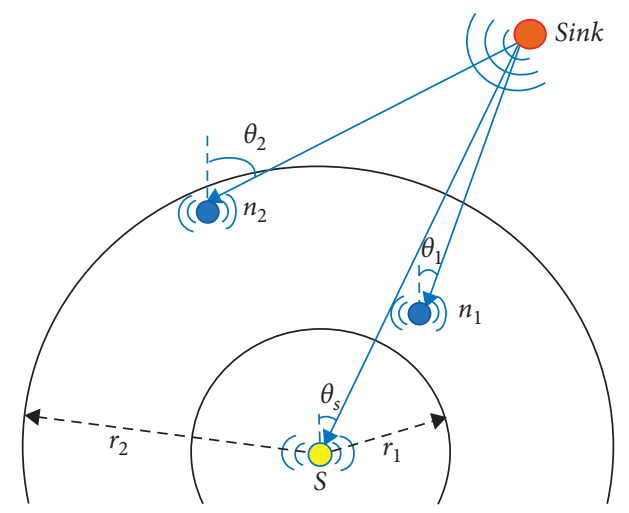

FIgURE 3: Two possible forwarders for node $S$ at power range $r 2$.

with residual energy indicators above 1 . In addition, it will select the forwarder with the least difference between the possible forwarder ackAoA and the sender sinkAoA. This ensures the minimum directional path toward the sink. The residual energy indicator in the forwarder table will be updated with the forwarders information of the received Ack packets. If the residual energy of all forwarders is very low (say at 1), the discovery procedure will be triggered to look for another set of possible forwarders using a higher transmission power level.

4.5. Power Control Implementation. To obtain the relative power values for the selected communication ranges used by the underwater nodes, we used the acoustics path loss model presented in $[45,46]$. The relation between the power level $P(l)$ values and the power spectral density (psd) of a transmitted acoustic signal $S_{l}(f)$ over the frequency bandwidth $B(l)$ is defined by the following:

$$
P(l)=\int_{B(l)} S_{l}(f) \mathrm{d} f .
$$

To simplify the calculation of the proposed protocol, we consider that $S_{l}(f)$ are flat over a narrow band bandwidth $B(l)$. So equation (3) becomes as follows:

$$
P(l)=B(l) S_{l}(f) .
$$

In order for a signal to be correctly received and detected by an acoustics modem, the signal-to-noise ratio $\operatorname{SNR}(l, f)$ should be greater than a modem specified threshold $\mathrm{SNR}_{0}$. This value is affected by the noise level $N(f)$, the attenuation level $A(l, f)$, and the psd of the signal level $S_{l}(f)$ that can be derived from equation (4). Therefore, $\operatorname{SNR}(l, f)$ at a distance $l$ and frequency $f$ can be written as follows:

$$
\operatorname{SNR}(l, f)=\frac{P(l)}{(N(f) A(l, f) B(l))} .
$$

The attenuation $A(l, f)$ can be obtained by applying equation (6) as follows:

$$
A(l, f)=10 \log \left(\frac{A(l, f)}{A_{0}}\right)=k .10 \log l+l .10 \log a(f),
$$


where $A_{0}$ is a unit normalizing factor that represents fixed losses, $a(f)$ is the absorption coefficient, and $k$ is the spreading factor defined as 1 for cylindrical, 2 for spherical, and 1.5 for practical spreading. The absorption coefficient is expressed using Thorp's formula that returns $a(f)$ in $\mathrm{dB} / \mathrm{km}$ for $f$ in $\mathrm{kHz}$ as follows:

$$
a(f)=\frac{0.11 f^{2}}{1+f^{2}}+\frac{44 f^{2}}{4100+f^{2}}+\frac{2.75 f^{2}}{1000+f^{2}}+0.003 .
$$

Additionally, the noise level $N(f)$ is a combination of different kind of noise such as the ambient noise which is caused by shipping $N_{s}(f)$, the waves noise which is caused by wind $N_{w}(f)$, the turbulence noise $N_{t}(f)$, and the thermal noise $N_{\text {th }}(f)$. Equations (8) to (11) listed below calculate each individual noise as follows:

$$
\begin{aligned}
10 \log _{10} N_{s}(f)= & 40+20(s-0.5)+26 \log _{10} f \\
& +60 \log _{10}(f+0.03), \\
10 \log _{10} N_{w}(f)= & 50+7.5 \sqrt{w}+20 \log _{10} f \\
& -40 \log _{10}(f+0.4), \\
10 \log _{10} N_{t}(f)= & 17-30 \log _{10} f, \\
10 \log _{10} N_{\text {th }}(f)= & -15+20 \log _{10} f .
\end{aligned}
$$

The $N(f)$ noise can now be computed in log scale by adding equations (8) to (11). It is worth noting that, for the deep marine environment, the $N(f)$ noise can be approximated as follows:

$$
10 \log _{10} N(f)=50-18 \log _{10} f .
$$

As we are interested in finding the required power level needed to be detected at a distance $l$, we can rewrite equation (4) in the following form:

$$
P(l)=\operatorname{SNR}(l, f) N(f) A(l, f) B(l) .
$$

The signal-to-noise ratio should be greater than an acoustic modem specified threshold $\mathrm{SNR}_{0}$ to detect and decode correctly. Substituting $S_{l}(f)$ from equation (13) into (4) gives the required acoustic power as follows:

$$
P(l) \approx \mathrm{SNR}_{0} B_{3}(l) \frac{N(f)}{A^{-1}(l, f)} .
$$

In acoustic communication, the transducer of an acoustic modem changes the electrical power $P t$ in watt to an acoustic sound power in micro-Pascal $(\mu \mathrm{Pa})$. The electrical power of an acoustic modem is a function of the modem efficiency and the acoustic power $P(l)$ needed to cover a distance $l$. Since no transducer is $100 \%$ efficient, the acoustic power is reduced by the transducer efficiency $\eta$. The relationship between the electrical power and the acoustic power needed to cover a distance $l$ is defined by the following:

$$
10 \log (\eta P t(l))=170.8-10 \log P(l)+\mathrm{DI},
$$

where 170.8 is a conversion factor between electric power and acoustic power. DI is the directivity index of the antenna, which is equal to zero for the omnidirectional antenna.

\section{Performance Evaluation}

To evaluate the proposed routing strategy, we have conducted extensive simulations for different static and mobile scenarios. In all simulation scenarios, the nodes are assumed to be uniformly distributed within $1 \mathrm{~km} \times 1 \mathrm{~km} \times 1 \mathrm{~km}$ three dimensional topology. We also adopted the power settings used in EvoLogic acoustic modem [47]. Nodes are able to set their transmission power Ptx to a set of values between 5.5 watt and 10.2 watt that is associated with a set of four communication ranges $250 \mathrm{~m}, 350 \mathrm{~m}, 500 \mathrm{~m}$, and $650 \mathrm{~m}$. Equation (14) is used to find the transmission power used at each range. The power value for receiving one bit of information has been set to $\operatorname{Prx}=0.8$ watt. The bit error ratio of acoustic modem has also been set to $10^{-9}$. The data packet size is 512 bits and the data rate is $30 \mathrm{kbps}$. The receiver minimum signal-to-noise ratio is $\mathrm{SNR}_{0}=30 \mathrm{~dB}$. The underlying MAC protocol has been used as the underwater broadcast MAC. We measured the average end-to-end delays of all packets generated throughout the whole simulation time which was fixed at 1200 s. The per bit energy consumption is defined by the total consumed power over the total number of delivered data bits. In addition, the total energy consumption is the sum of all energy dissipated by all nodes. The delivery ratio is calculated as the total number of packets submitted successfully to the sink node over the total number of packets generated by all source nodes.

5.1. Static Scenarios. Here, we evaluate the performance of the proposed directional selective power protocol. A comparison between the performance of the proposed protocol and that of the VDBR protocol is performed. We also compare the performance of the proposed protocol with that of the other two versions of a directional fixed power routing using two ranges ( $500 \mathrm{~m}$ and $250 \mathrm{~m}$ ). The two aforementioned ranges represent two cases where a low range at $250 \mathrm{~m}$ is suitable for dense networks, and the $500 \mathrm{~m}$ works best to maintain connectivity for the sparse networks. Figure 4 shows the delivery ratio for the fixed directional routing at the range of $250 \mathrm{~m}$ (DR250). Here, one can see that the delivery ratio is between $20 \%$ and $45 \%$. In addition, the achieved ration almost doubled to that of RD250 when increasing the power range to $500 \mathrm{~m}$ (DR500). A fixed low communication range (i.e., DR250) requires a dense network to maintain the network connectivity and hence achieves a high delivery ratio. However, the VDBR and DSPR protocols have a very high delivery ratio above $92 \%$ for all node densities. Furthermore, the VDBR protocol achieves a slightly better delivery ratio than the DSPR one. This is due to the nature of the depth based routing, which allows multiple forwarders at each step to acquire higher delivery probability. As a result, the power consumption is drastically increased. 


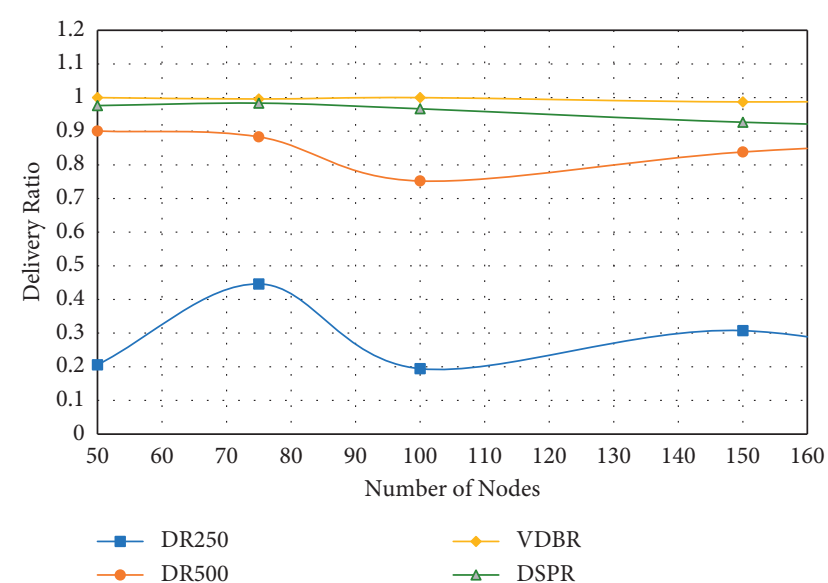

FIgure 4: Delivery ratio, one sink, static scenario.

Figure 5 shows the end-to-end delays for one sink with varying nodes density. The data packets in the DBR250 and VDBR reach the surface sinks in less time than that of DR500 and DSPR by about 0.2 seconds. This is due to the fact that the depth based routing protocols do not employ retransmissions for dropped packets and hence data is delivered faster with no delays. In the DR250, the delivery is scarce, and it happens only when a sender finds one forwarder within $250 \mathrm{~m}$. Therefore, the probability of collisions that triggers the time-consuming retransmissions in DR500 is lowered in DR250. In the DSPR, the extra delay is due to the time needed to exchange the control packet for the forwarder and the power range selection. As a result, for the time sensitive applications, the VDBR protocol is rather to be used in favour of the DSPR protocol.

Although the VDBR protocol shows a relatively low endto-end delay and high delivery ratio, the energy expenditure is extremely high. Table 2 shows that the energy consumption by the VDBR protocol is around 8 times higher than that of the DSPR protocol when the number of nodes is 50. Moreover, the energy consumption is 15.7 times higher than that at 200 nodes. This excessive energy expenditure is due to the flooding of multiple copies of the same packet when the number of nodes in the network increases. In the directional scenarios, we limit the maximum number of nodes that can participate in the forwarding process to two nodes. Each node keeps a record of the forwarded packet IDs to forbid forwarding the same packet twice. Thus, the unnecessary forwarding is reduced in the DBR protocol.

Figure 6 also shows that using the selective power routing achieves considerably better energy savings than that of both fixed case scenarios at the $250 \mathrm{~m}$ range for both high and low node densities with more than $40 \%$ savings. The per bit energy saving achieved by the DSPR protocol is more than $25 \%$ compared to the DR500 when the node densities get high.

The proposed routing protocol has also been illustrated with four sinks. Similarly, the proposed protocol achieves a noticeable enhancement in both energy and delivery ratios. Figure 7 shows that the DR500, VDBR, and DSPR protocols delivery ratios are always above 90\%. However, the DR250

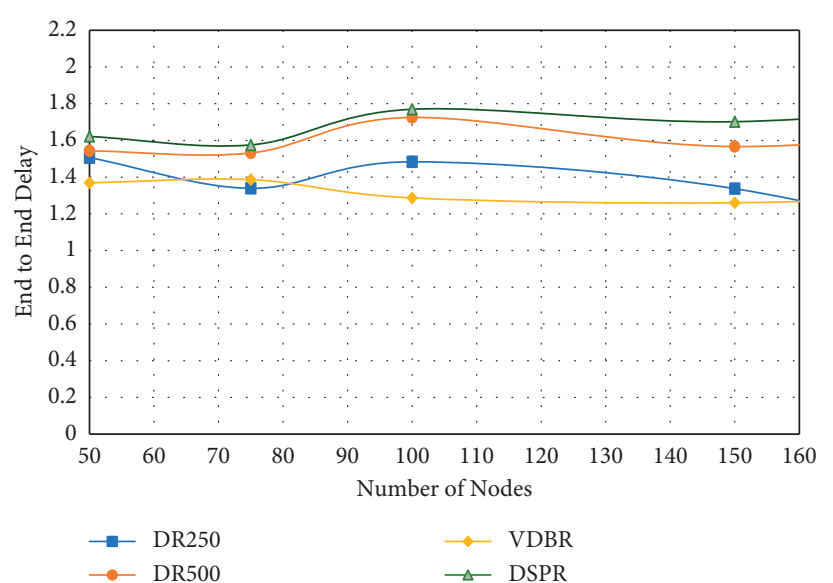

Figure 5: The end-to-end delay, one sink, static scenario.

TABLe 2: Per bit energy consumption VDBR vs DSPR.

\begin{tabular}{lccc}
\hline & \multicolumn{2}{c}{ Per bit energy $10^{-3}$ joule } & \\
Routing scheme & at 50 node & at 100 node & at 200 node \\
\hline VDBR & 18.50 & 32.47 & 51.04 \\
DSPR & 2.22 & 2.44 & 3.23 \\
\hline
\end{tabular}

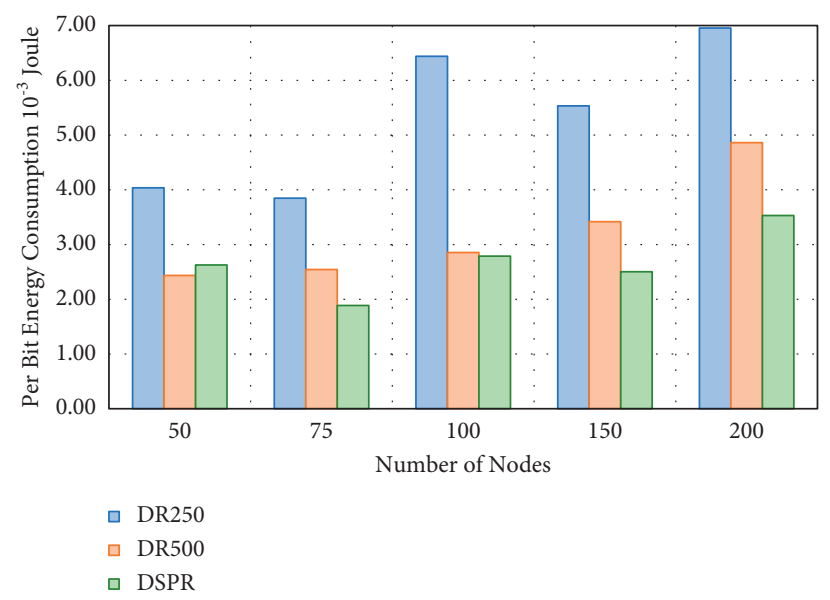

FIgure 6: Per bit energy consumption, one sink, static scenario.

protocol achieves a very low delivery ratio. This is because of the limited communication range where the senders can rarely find forwarders. When we increase the number of nodes (i.e., higher nodes densities), the probability of finding forwarders increases, and hence the delivery ratio increases to $46 \%$ at 150 nodes.

Figure 8 shows the end-to-end delay with four sinks. One can see that the end-to-end delay is slightly less than that for one sink architecture. This is because having more sinks will lead to shortening the distances from the underwater nodes to the surface and thus reducing the propagation delays. The other forms of delay are a result of control message exchange and packet retransmissions that slows the directional routing protocol compared to the VDBR. 


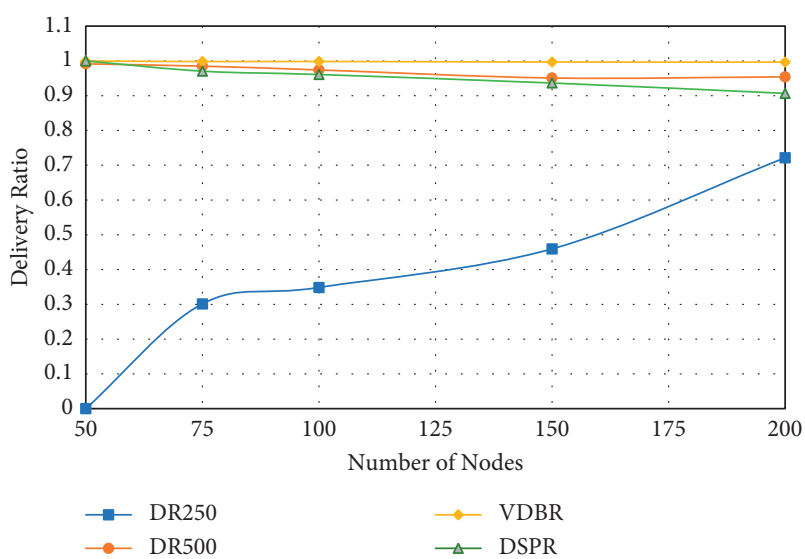

Figure 7: Delivery ratio, four sinks, static scenario.

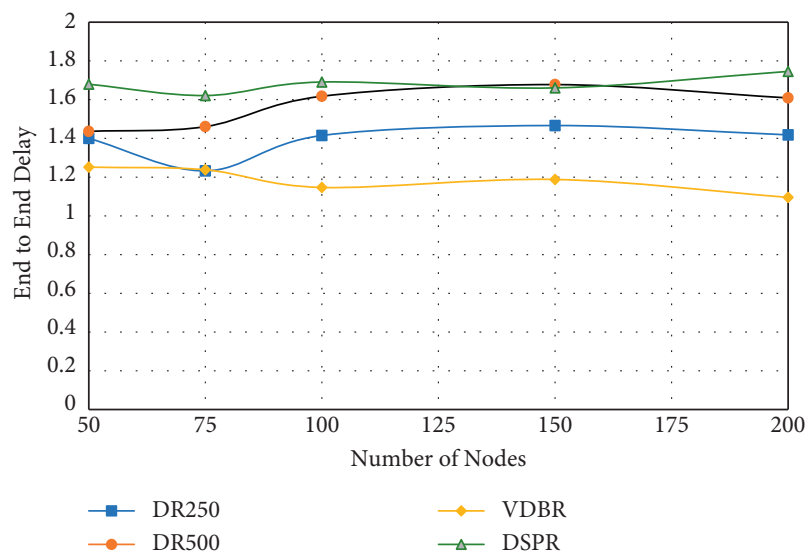

Figure 8: The end-to-end delay, four sinks, static scenario.

The energy expenditure is reduced when using multiple sinks architecture as shown in Figure 9. DSPR is also shown to outperforms both fixed directional routing techniques at DR250 and DR500. More than 20\% saving is obtained when number of nodes greater than 150 . This results from the fact that in DSPR, nodes tends to use lowest power level needed to reach forwarders. At low densities energy saving in DR500 is almost the same as in DSPR since both strategies tend to use the same power range at $500 \mathrm{~m}$ and hence will consume same amount of power.

5.2. Mobility Consideration. We ran a set of more experiments to test our technique against directional routing in dynamic scenarios. For modeling the node mobility, we adopted the a tidal mobility model where tidal and residual current fields are used for determining the composite current field. The residual current field is assumed to be an infinite sequence of clockwise and anticlockwise spinning eddies, and the tidal field is assumed to be a spatially uniform oscillating current in one direction [23]. In this model, the velocity fields in the $x$ and $y$ directions field are approximated by using the kinematic model as follows:

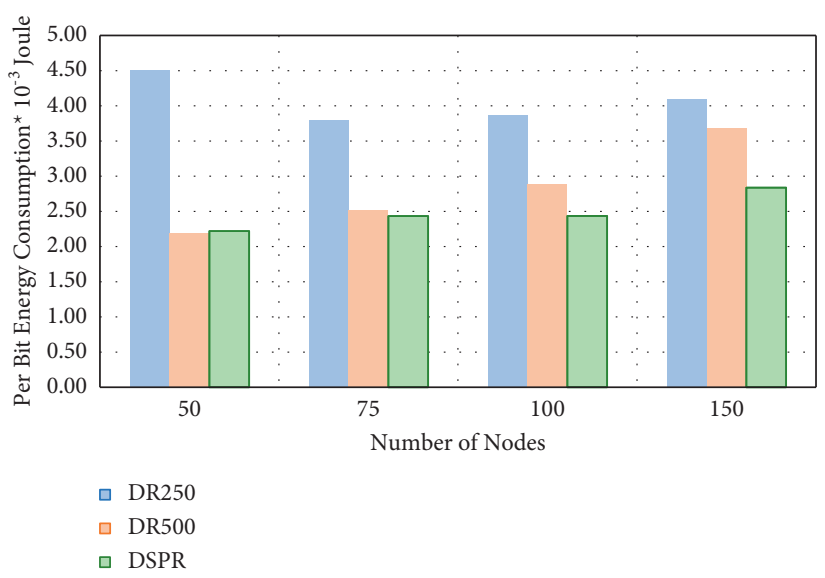

FIgURE 9: Per bit energy consumption, four sinks, static scenario.

$$
\begin{aligned}
& V_{x}=k_{1} \lambda v \sin e\left(k_{2} x\right) \cos \left(k_{3 y}\right)+k_{1} \lambda \cos \left(2 k_{1} t\right)+k_{4}, \\
& V_{y}=-\lambda v \cos \left(k_{2} x\right) \sin \left(k_{3} y\right)+k_{5},
\end{aligned}
$$

where $V_{x}$ and $V_{x}$ are the speed in the $x$ and $y$ directions. $k_{1}$, $k_{2}, k_{3}, k_{5}$ and $\lambda$ are parameters related to water environment factors including bathymetry and tides. In our simulations, we used the settings for deep underwater environments as in [22] with an underwater node speed in the range of $1-5 \mathrm{~m} / \mathrm{s}$. In DR250 and due to limited communication range, senders can rarely find forwarders at low node densities. In fixed scenarios delivery ratio is less than $46 \%$ when the number of nodes is less than 150. When the network is mobile, forwarders keep moving and get out of senders' range. Senders with fixed low power range will suffer updating their forwarding list and the delivery ration will drop more. Figure 10 shows that for a mobile network, the delivery ratio in fixed directional power cases is less than $10 \%$ at the $250 \mathrm{~m}$ range and is less than $68 \%$ when using the $500 \mathrm{~m}$ range. On the other hand, the delivery ratio in DSPR is just slightly lower than that found in a static network situation and remains over $90 \%$ when varying node density. As a result, DSPR is best suited for mobile scenarios since it improves network connectivity significantly compared to fixed directional routing strategies.

The energy consumption is also advantageous in the DSPR protocol with more than $80 \%$ and $30 \%$ saving compared to the DR250 and the DR500, respectively, as shown in Figure 11. The extra energy expenditure in the DR250 protocol results from increasing the number of hops. Thus, more nodes use lower power levels to participate in the data packet forwarding. In the DR500 protocol, the collision domain is high and the extra energy is consumed because of the retransmissions. Therefore, selecting the best power level shows an advantage over the other two variations of directional routing. This is because the usage of the correct power level is chosen in accordance with forwarders availability.

The advantage of DSPR over other tested static strategies and the VDBR was also clear when finding total energy consumption by nodes. Our simulation results showed a much higher energy expenditure of dynamic case VDBR 


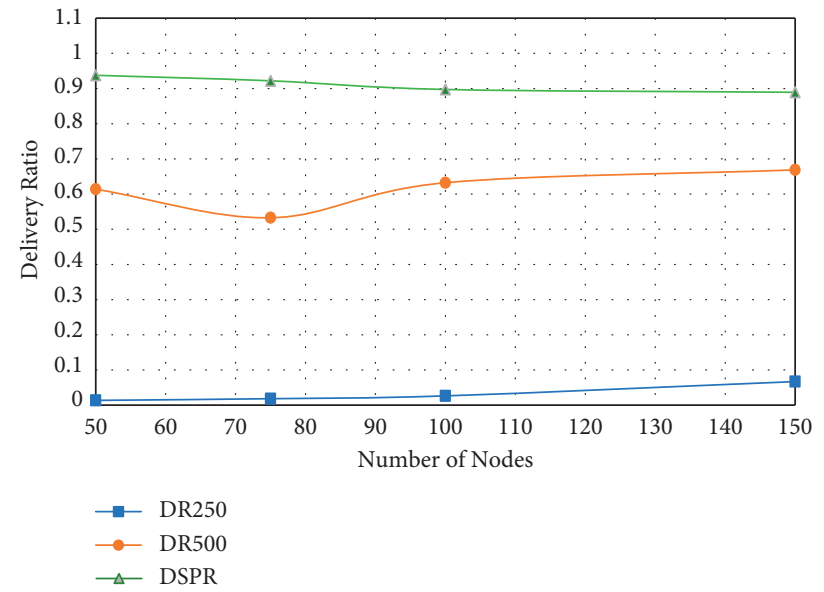

FIgURE 10: Delivery ratio, mobile scenario.

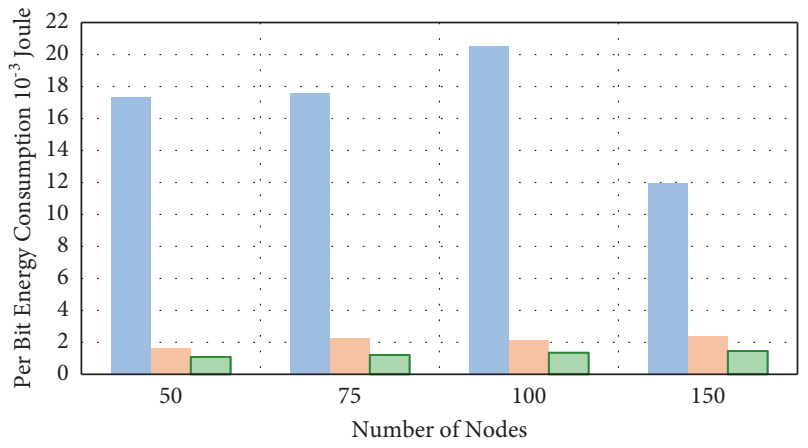
$\square$ DR250
$\square$ DR500
$\square$ DSPR

FIgURe 11: Per bit energy consumption, mobile scenario.

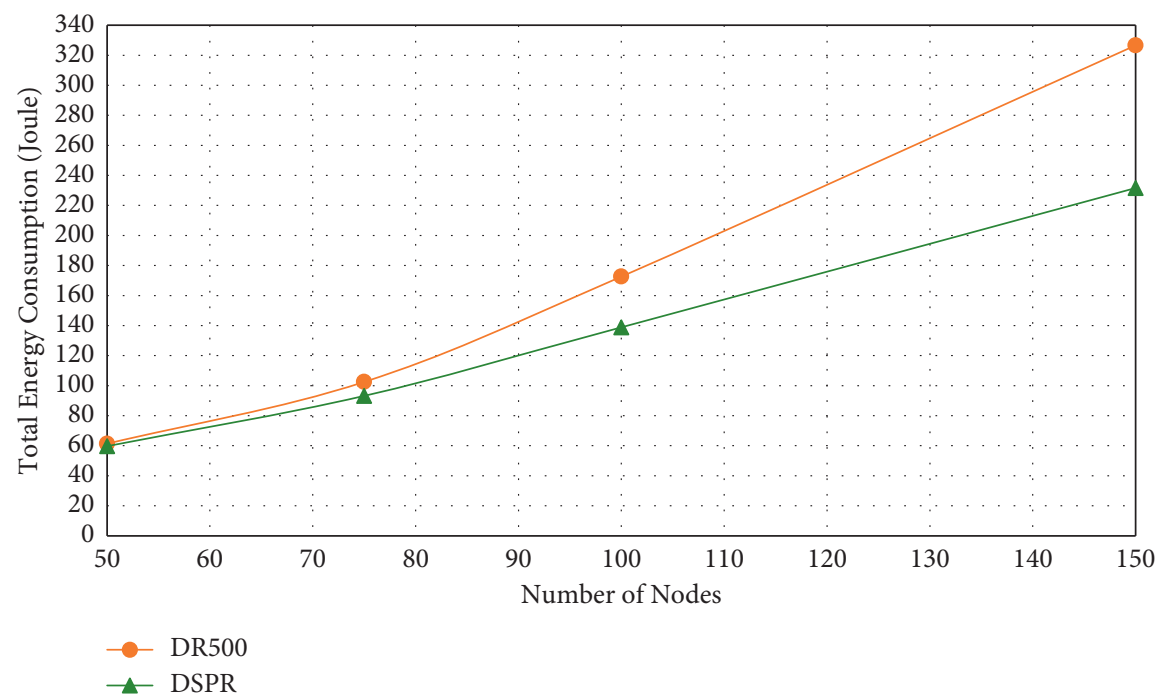

FIgURE 12: Total energy consumption, mobile scenario. 
than that for the static case VDBR as shown previously in Table 2. The energy expenditure, in this case, was at least 20 times more than the dynamic case of our proposed DSPR. On the other hand, very low energy consumption was found with fixed directional routing DR250. This is due to the fact no energy is consumed in sending data packets as forwarders are not actually found at this low range. Therefore, in this case, we only compare DSPR with DR500 since they tend to have a comparable delivery ratio. Figure 12 shows that the total energy expenditure is reduced when the network becomes denser as nodes tend to use lower power levels to reach forwarders. Therefore, collisions and retransmissions are reduced and the network lifetime in enhanced.

\section{Conclusion}

In this paper, we focused our attention on the issues and challenges that affect the protocol design for the underwater part of IoUT network. Increasing reliability by maintaining connectivity and conserving energy are of the most important requirements for efficient communication in a dynamic underwater environment. The unique challenges imposed by the characteristics of the underwater communication channel have impacted the underwater routing performance. To overcome these challenges, we proposed a directional selective power technique that is location-free, robust to mobility, and energy efficient. In the proposed technique, we acquire the simplicity of sender-based angle of arrival forwarding decision with a practical power control implementation of an acoustic modem. The senders in the proposed protocol select the best forwarder in the direction of the nearest sink via directional routing. In addition, the sender nodes can switch to use a higher power level when the network is sparse. This will help the acoustic signal to traverse higher distances to locate the most suited forwarders. Hence, a forwarder is guaranteed to be found with the least possible power level to reduce the energy expenditure and maintain network connectivity. Extensive simulations show enhancements over other protocols regarding energy expenditure and delivery ratio for both static and mobile scenarios, dense or sparse networks, and with one or multiple surface sink architectures. However, a slight increase in the end-to-end delays is observed as an overhead of switching to the correct power level and increasing the number of hops. In future work, an optimization of the number of power levels is to be conducted to further reduce energy expenditure and to avoid transmission delay overhead.

\section{Data Availability}

The data used to support the findings of this study are included within the article.

\section{Conflicts of Interest}

The authors declare that they have no conflicts of interest.

\section{References}

[1] C.-C. Kao, Y.-S. Lin, G.-D. Wu, and C.-J. Huang, "A comprehensive study on the internet of underwater things: applications, challenges, and channel models," Sensors, vol. 17, no. 7, p. 1477, 2017.

[2] D. Anguita, D. Brizzolara, and G. Parodi, "Building an underwater wireless sensor network based on optical: communication: research challenges and current results," in Proceedings of the 2009 Third International Conference on Sensor Technologies and Applications, Athens, Greece, June 2009.

[3] L. Liu, S. Zhou, and J.-H. Cui, "Prospects and problems of wireless communication for underwater sensor networks. wiley wcmc special issue on underwater sensor networks," Wireless Communications and Mobile Computing, vol. 8, pp. 977-994, 2008.

[4] M. C. Domingo, "Magnetic induction for underwater wireless communication networks," IEEE Transactions on Antennas and Propagation, vol. 60, no. 6, pp. 2929-2939, 2012.

[5] D. E. Lucani, M. Stojanovic, and M. Medard, "On the relationship between transmission power and capacity of an underwater acoustic communication channel," in Proceedings of the OCEANS 2008 - MTS/IEEE Kobe Techno-Ocean, April 2008.

[6] G. Tuna and V. C. Gungor, "A survey on deployment techniques, localization algorithms, and research challenges for underwater acoustic sensor networks," International Journal of Communication Systems, vol. 30, no. 17, Article ID e3350, 2017.

[7] P. Xu, B. Yan, and S. Hu, "DOA estimation of multiple sources in sparse space with an extended array technique," Cluster Computing, vol. 19, no. 3, pp. 1437-1447, 2016.

[8] M. Li, X. Du, X. Liu, and C. Li, "Shortest path routing protocol based on the vertical angle for underwater acoustic networks," Journal of Sensors, vol. 2019, pp. 1-14, 2019.

[9] M. A. Bzoor, Y. Zhu, J. Liu, R. Ammar, J. H. Cui, and S. Rajasekaran, "An adaptive power controlled routing protocol for underwater sensor network," International Journal of Sensor Networks, vol. 18, no. 3/4, pp. 238-249, 2015.

[10] Z. Fang, J. Wang, J. Du, X. Hou, Y. Ren, and Z. Han, "Stochastic optimization aided energy-efficient information collection in internet of underwater things networks," IEEE Internet of Things Journal, vol. 9, no. 3, pp. 1775-1789, 2022.

[11] Z. Hong, X. Pan, P. Chen, X. Su, N. Wang, and W. Lu, "A topology control with energy balance in underwater wireless sensor networks for iot-based application," Sensors, vol. 18, no. 7, p. 2306, 2018.

[12] M. Barbeau, J. Garcia-Alfaro, E. Kranakis, and S. Porretta, "The sound of communication in underwater acoustic sensor networks," in Ad Hoc Networks, pp. 13-23, Springer International Publishing, Berlin, Germany, 2018.

[13] Z. Fang, J. Wang, C. Jiang, Q. Zhang, and Y. Ren, “AoI-inspired collaborative information collection for AUV-assisted internet of underwater things," IEEE Internet of Things Journal, vol. 8, no. 19, pp. 14559-14571, 2021.

[14] N. Iruthayanathan, V. K. Srinivasan, V. Rajendran, and R. Palanimurugan, "Mitigating ambient noise and multipath propagation in underwater communication using the DSTTD-OFDM system," Computers \& Electrical Engineering, vol. 53, pp. 409-417, 2016.

[15] D. Pompili, T. Melodia, and I. F. Akyildiz, "Deployment analysis in underwater acoustic wireless sensor networks," in Proceedings of the 1st ACM International Workshop on Underwater Networks, ser. WUWNet '06, ACM International Workshop on Underwater Networks, pp. 48-55, Association for Computing Machinery, New York, NY, USA, 2006.

[16] S. Ibrahim, M. Al-Bzoor, J. Liu, R. Ammar, S. Rajasekaran, and J.-H. Cui, "General optimization framework for surface 
gateway deployment problem in underwater sensor networks," EURASIP Journal on Wireless Communications and Networking, vol. 2013, no. 1, p. 128, 2013.

[17] B. Liu, F. Ren, C. Lin, Y. Yang, R. Zeng, and H. Wen, "The redeployment issue in underwater sensor networks," in Proceedings of the Global Telecommunications Conference, 2008. IEEE GLOBECOM 2008, pp. 1-6, IEEE, New Orleans, LA, USA, December 2008.

[18] J. Liu, W. Guan, G. Han, J. H. Cui, L. Fiondella, and M. AlBzoor, "A dynamic surface gateway placement scheme for mobile underwater networks," Sensors, vol. 19, no. 9, 2019.

[19] A. Serhani, N. Naja, and A. Jamali, "AQ-routing: mobility-, stability-aware adaptive routing protocol for data routing in MANET-IoT systems," Cluster Computing, vol. 23, no. 1, pp. 13-27, 2020.

[20] S. Beerens, H. Ridderinkhof, and J. Zimmerman, "An analytical study of chaotic stirring in tidal areas," Chaos, Solitons \& Fractals, vol. 4, no. 6, pp. 1011-1029, 1994.

[21] A. Caruso, F. Paparella, L. F. M. Vieira, M. Erol, and M. Gerla, "The meandering current mobility model and its impact on underwater mobile sensor networks," in Proceedings of the IEEE INFOCOM 2008 - The 27th Conference on Computer Communications, ser. IEEE INFOCOM 2008, The 27th Conference on Computer Communications, pp. 221-225, IEEE, Phoenix, AZ, USA, April 2008.

[22] Z. Zhou, Z. Peng, J.-H. Cui, Z. Shi, and A. Bagtzoglou, "Scalable localization with mobility prediction for underwater sensor networks," IEEE Transactions on Mobile Computing, vol. 10, no. 3, pp. 335-348, 2011.

[23] Y. Zhang, J. Liang, S. Jiang, and W. Chen, “A localization method for underwater wireless sensor networks based on mobility prediction and particle swarm optimization algorithms," Sensors, vol. 16, no. 2, p. 212, 2016.

[24] M. Dong, H. Li, R. Yin, Y. Qin, and Y. Hu, "Scalable asynchronous localization algorithm with mobility prediction for underwater wireless sensor networks," Chaos, Solitons \& Fractals, vol. 143, Article ID 110588, 2021.

[25] G. J. Alqahtani and F. Bouabdallah, "Energy-efficient mobility prediction routing protocol for freely floating underwater acoustic sensor networks," Frontiers in Communications and Networks, vol. 2, 2021.

[26] Z. Wan, S. Liu, W. Ni, and Z. Xu, “An energy-efficient multilevel adaptive clustering routing algorithm for underwater wireless sensor networks," Cluster Computing, vol. 22, no. S6, pp. 14651-14660, 2019.

[27] R. Coutinho and A. Boukerche, "Data collection in underwater wireless sensor networks: research challenges and potential approaches," in Proceedings of the 20th ACM International Conference on Modelling, Analysis and Simulation of Wireless and Mobile Systems, vol. 11, pp. 5-8, Miami, FL, USA, 2017.

[28] M. Jouhari, K. Ibrahimi, H. Tembine, and J. Ben-Othman, "Underwater wireless sensor networks: a survey on enabling technologies, localization protocols, and internet of underwater things," IEEE Access, vol. 7, pp. 96879-96899, 2019.

[29] K. M. Awan, P. A. Shah, K. Iqbal, S. Gillani, W. Ahmad, and Y. Nam, "Underwater wireless sensor networks: a review of recent issues and challenges," Wireless Communications and Mobile Computing, vol. 2019, Article ID 6470359, 20 pages, 2019.

[30] H. Yan, Z. J. Shi, and J.-H. Cui, "Dbr: depth-based routing for underwater sensor networks," in Networking 2008 Ad Hoc and Sensor Networks, Wireless Networks, Next Generation Internet, ser. Lecture Notes Computer Science, vol. 4982, pp. 72-86, Springer, Berlin, Heidelberg, 2008.
[31] A. Wahid, S. Lee, H.-J. Jeong, and D. Kim, "EEDBR: energyefficient depth-based routing protocol for underwater wireless sensor networks," in Communications in Computer and Information Science, pp. 223-234, Springer, Berlin, Heidelberg, 2011.

[32] B. Diao, Y. Xu, Z. An, F. Wang, and C. Li, "Improving both energy and time efficiency of depth-based routing for underwater sensor networks," International Journal of Distributed Sensor Networks, vol. 2015, Article ID 781932, 9 pages, 2015.

[33] M. Jafri, S. Ahmed, N. Javaid, Z. Ahmad, and R. Qureshi, "Adaptive mobility of courier nodes in threshold-optimized DBR protocol for underwater wireless sensor networks," in Proceedings of the 2013 Eighth International Conference on Broadband and Wireless Computing, Communication and Applications, October 2013.

[34] N. Javaid, M. R. Jafri, Z. A. Khan, U. Qasim, T. A. Alghamdi, and M. Ali, "iAMCTD: improved adaptive mobility of courier nodes in threshold-optimized DBR protocol for underwater wireless sensor networks," International Journal of Distributed Sensor Networks, vol. 10, no. 11, Article ID 213012, 2014.

[35] N. Javaid, M. R. Jafri, S. Ahmed et al., "Delay-sensitive routing schemes for underwater acoustic sensor networks," International Journal of Distributed Sensor Networks, vol. 11, no. 3, Article ID 532676, 2015.

[36] Z. Zhou, B. Yao, R. Xing, L. Shu, and S. Bu, "E-carp: an energy efficient routing protocol for uwsns in the internet of underwater things," IEEE Sensors Journal, vol. 16, no. 11, pp. 4072-4082, 2016.

[37] S. Basagni, C. Petrioli, R. Petroccia, and D. Spaccini, "CARP: a Channel-aware routing protocol for underwater acoustic wireless networks," Ad Hoc Networks, vol. 34, pp. 92-104, 2015.

[38] A. Khan, I. Ahmedy, M. Anisi et al., "A localization-free interference and energy holes minimization routing for underwater wireless sensor networks," Sensors, vol. 18, no. 2, p. 165, 2018.

[39] N. Usman, O. Alfandi, S. Usman et al., "An energy efficient routing approach for IoT enabled underwater WSNs in smart cities," Sensors, vol. 20, no. 15, p. 4116, 2020.

[40] A. Celik, N. Saeed, B. Shihada, T. Y. Al-Naffouri, and M.-S. Alouini, "Opportunistic routing for opto-acoustic internet of underwater things," IEEE Internet of Things Journal, vol. 9, no. 3, pp. 2165-2179, 2022.

[41] M. Al-Bzoor, T. Gharaibeh, and O. Ai-Ta'ani, “Avoiding routing voids with selective transmission power in underwater wireless sensor networks," in Proceedings of the 2021 IEEE Jordan International Joint Conference on Electrical Engineering and Information Technology (JEEIT), November 2022.

[42] M. R. Jafri, M. M. Sandhu, K. Latif, Z. A. Khan, A. U. H. Yasar, and N. Javaid, "Towards delay-sensitive routing in underwater wireless sensor networks," Procedia Computer Science, vol. 37, pp. 228-235, 2014.

[43] A. Umar, N. Javaid, A. Ahmad et al., "DEADS: depth and energy aware dominating set based algorithm for cooperative routing along with sink mobility in underwater WSNs," Sensors, vol. 15, no. 6, pp. 14458-14486, 2015.

[44] E. Dubrovinskaya, V. Kebkal, O. Kebkal, K. Kebkal, and P. Casari, "Underwater localization via wideband directionof-arrival estimation using acoustic arrays of arbitrary shape," Sensors, vol. 20, no. 14, p. 3862, 2020.

[45] M. Stojanovic, "On the relationship between capacity and distance in an underwater acoustic communication channel," ACM Sigmobile-Mobile Computing and Communications Review, vol. 11, no. 4, pp. 34-43, 2007. 
[46] H. U. Yildiz, V. C. Gungor, and B. Tavli, "Packet size optimization for lifetime maximization in underwater acoustic sensor networks," IEEE Transactions on Industrial Informatics, vol. 15, no. 2, pp. 719-729, 2019.

[47] Evologic, "Evologics underwater acoustic modems," 2021, https://evologics.de/acoustic-modems. 